Part VIII

RADIO STRUCTURE OF AN ACTIVE REGION 


\title{
HOMOLOGY OF SOLAR RADIO EVENTS
}

\author{
A. D. FOKKER \\ (Sterrewacht 'Sonnenborgh', Utrecht, The Netherlands)
}

\begin{abstract}
A BSTRACT
Successive flares within the same centre of activity sometimes produce radio events that are remarkably similar. The occurrence of such homologous radio events is commonly restricted to periods of less than $\mathbf{4 8}$ hours.

Typical for certain centres of activity is their tendency to give rise to impulsive microwave bursts. Such bursts may occur repeatedly during a period of more than a week. The distributions of the lengths of periods during which series of homologous bursts and of impulsive microwave bursts occur are compared.
\end{abstract}

Ellison et al. (1960) called attention to the occurrence of homologous flares. These are flares which occur successively in the same active region, correspond in position relative to the local sunspots, and show a common pattern of structure and development.

The concept of homology can be extended to the flare-associated radio events, since occasionally two or more flares in the same centre of activity give rise to radio events that are remarkably similar in one or more of the main frequency bands: centimetric, decimetric and metric. Sometimes the intensity curves of two radio events show detailed similarity over a wide range of frequencies. But even if the intensity curves do not resemble each other in detail, two radio events may be similar in that their responses in the various frequency bands have comparable durations and intensities. On the basis of the correspondence of intensity and duration it is possible to select pairs of homologous radio events in the tabulations of distinctive events in the Quarterly Bulletin on Solar Activity. On applying a certain system of rating the degree of correspondence of duration and intensity in the various frequency bands, it is possible to derive a quantitative measure for the extent to which two radio events are homologous. We determined the measure of homology for several pairs of radio events that produced responses in each of the $\mathrm{cm}$, $\mathrm{dm}$, and $\mathrm{m}$ frequency bands. Two events were considered as homologous if this measure surpassed a certain value.

Among 69 pairs of events that occurred in the same centre of activity during the period 1959-61, there were 14 homologous pairs, or $20 \%$; among 83 pairs of events taken arbitrarily from different centres of activity, only 4 , or $5 \%$ were homologous.

We thus conclude that there is a significant tendency for homologous radio events to occur within one centre of activity.

Kiepenheuer (ed.), Structure and Development of Solar Active Regions, 553-555. C I.A.U. 

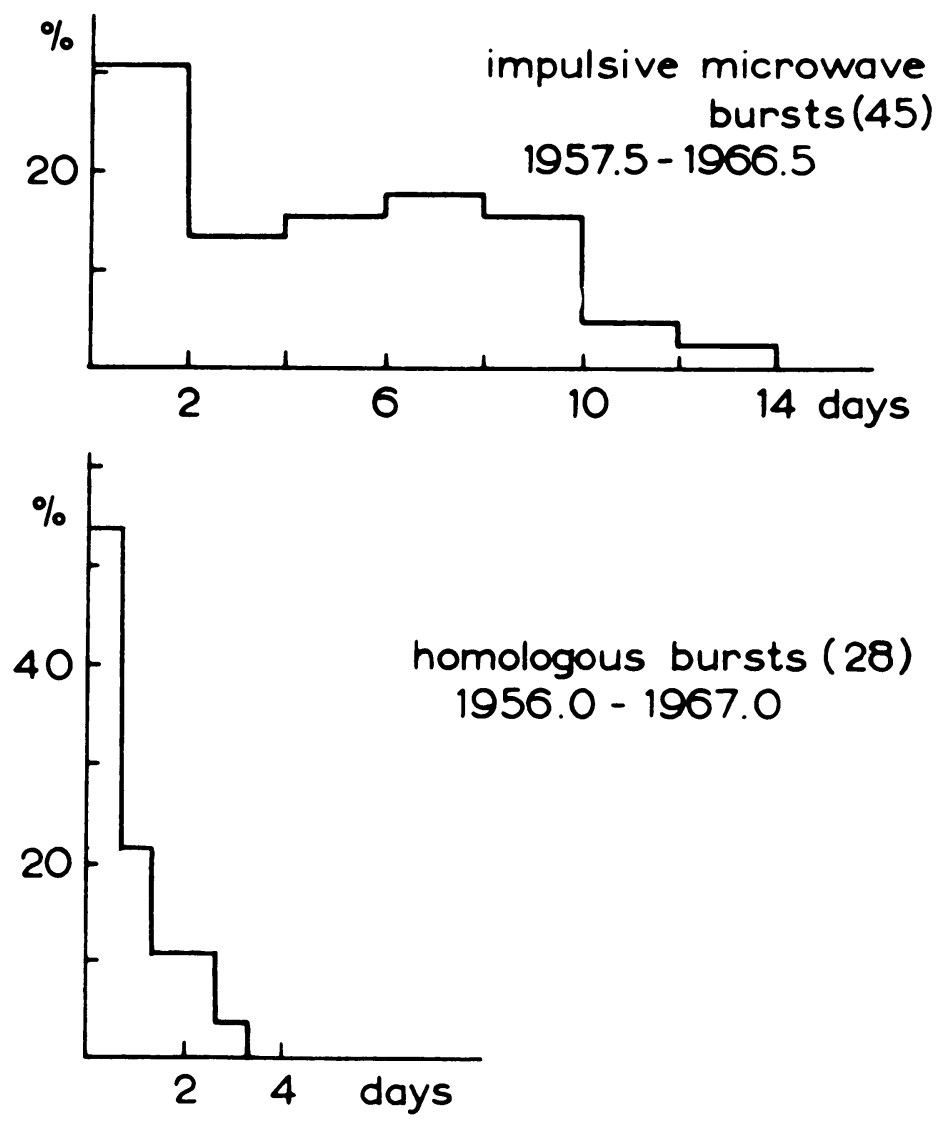

FiG. 1. Histograms of time intervals between the first and the last events that belong to a series of impulsive microwave bursts and of homologous radio events, respectively.

In a centre of activity homologous events succeed each other at time intervals which seldom surpass 48 hours. A histogram of such time intervals is given in the lower part of Figure 1.

It is interesting to draw into comparison the occurrence, within one centre of activity, of the well-defined class of impulsive microwave bursts. The impulsiveness of a burst can be determined quantitatively as the steepness of the rise of intensity. If this figure surpasses a certain value, the microwave burst may be considered an impulsive one.

Impulsive microwave bursts need not at all be homologous: their intensity, duration and detailed intensity curves may be quite different. Fokker and Roosen (1961) showed that the impulsiveness of microwave bursts is a very characteristic feature of bursts that occur in specific centres of activity. Contrary to the homology, the impulsiveness of microwave bursts may be preserved during several days in succession. 
A histogram of time intervals during which impulsive microwave bursts occurred associated with flares in one and the same centre of activity is given in the upper part of Figure 1.

There is an outstanding difference between the two histograms in Figure 1. The type of condition which gives rise to the occurrence of homologous radio events apparently does not persist for much longer than 2 days. On the other hand, the impulsiveness of microwave bursts is governed by situations that may persist for longer than a week.

We can only guess what the factors may be that govern the occurrence of a particular type of radio event. The impulsiveness may, for instance, be related to the existence of strong gradients in the magnetic field near the place of origin of the flare. Homology perhaps occurs only if the detailed structure of the magnetic field remains unchanged and if the acceleration of particles takes place in exactly the same location.

There is a suggestion that the histogram for the impulsive microwave bursts consists of two components. It will be of interest to see whether this feature is preserved as the available material increases. If genuine, the effect would seem to imply that there exist two conditions of a different nature, and both may give rise to an impulsive microwave burst.

\section{References}

Ellison, M.A., McKenna, S. M.P., Reid, J.H. (1960) Dunsink Obs. Publ., 1, No. 1.

Fokker, A.D., Roosen, J. (1961) Bull. astron. Inst. Netherl., 16, 83.

\section{DISCUSSION}

De Jager: Your histogram of impulsive microwave bursts extends over 14 days, which is the maximum observable time of an active region. Did you correct your observations for the fact that at least part of the observations extended over a time interval $<14$ days?

Fokker: No corrections were made. 